\title{
Effective permittivity of co-evaporated metal-organic mixed films
}

\author{
Andreas Mischok ${ }^{1}$, Nathan Hale ${ }^{1}$, Malte C. Gather ${ }^{1,2}$, Andrea di Falco ${ }^{1}$ \\ ${ }^{1}$ School of Physics and Astronomy, University of St. Andrews, North Haugh, St. Andrews \\ KY16 9SS, United Kingdom \\ ${ }^{2}$ Centre for Nanobiophotonics, Department of Chemistry, University of Cologne, Greinstr. 4- \\ 6, 50939 Köln, Germany
}

\begin{abstract}
The combination of organics and metals in a composite film holds promise for combining plasmonic interaction with gain and for the realization of epsilon-near-zero (ENZ) metamaterials. In particular, fluorescent organic dyes can be used to compensate the plasmonic losses of a homogenized metal-organic material. Here, we fabricate such films through thermal co-evaporation of silver and an organic host:guest system and investigate experimentally the resulting linear optical properties for varying metal concentrations. We extract the effective permittivity of the resulting films with ellipsometry measurements and demonstrate the formation of silver nanoparticles and resulting strong localised surface plasmon resonances, up until a percolation threshold. Through enhanced light-matter interaction, we observe a maximum of the photoluminescence for a concentration of $15 \%$ in volume of metal in the composite material. These results showcase a variety of growth parameters and will be useful for the future design of gain-compensated plasmonics and ENZ metamaterials.
\end{abstract}

\section{Introduction}

Plasmonic resonances enable strong light-matter interaction through light localization at the nanoscale and large field enhancement. However, these attractive features are unavoidably accompanied by strong Ohmic losses, associated with the oscillation of the electron clouds in the metal. Some applications exploit the strong absorption of light at visible wavelengths, e.g. for sensing ${ }^{1}$ or structured coloring applications ${ }^{2}$. However, for applications that are affected by power or energy budgets or localized heating limitations, plasmonic losses can be very detrimental. An effective solution aims at reducing the interaction in time and space of the optical field with the metal, at the cost of increasing the modal volume and reducing the field enhancement, as is the case for e.g. long range surface plasmons ${ }^{3,4}$. An alternative strategy uses optical gain to compensate for the losses, e.g. bringing in close proximity with the metals suitable active media, like dyes, quantum dots or semiconductor materials ${ }^{5-9}$. This strategy presents a series of challenges, typically associated with the need of using short optical pump pulses or of dealing with very high injection currents. However, with suitable arrangements, the gain can be sufficient to support lasing or net optical amplification ${ }^{10-13}$. Recent reviews address carefully the many issues and challenges associated with the realization of spasers $^{14,15}$, typically involving engineering the modal volume and the radiation channels, through careful fabrication of tailored nanostructures.

However, if the aim is to reduce the losses, rather than eliminating them completely, the requirements can be relaxed. An interesting example is a class of artificial metamaterials, with subwavelength structure and homogenized response. In this case, the aim is to reduce the imaginary component of the effective permittivity. This goal is particularly critical for materials with vanishing real part of the effective permittivity. For contained losses, epsilon near zero 
(ENZ) metamaterials exhibit extremely low refractive indices (making them index-near-zero, INZ), with remarkable effect on light propagation, imaging and light-matter interaction enhancement ${ }^{16-21}$. To date, several promising strategies have been put forward to reduce the absolute value of the effective refractive index, mostly based on the hybridisation of organic dyes with the metallic layers, particles or nanoshells ${ }^{22-24}$. Organic gain materials are particularly attractive from a technological point of view because of the relative ease of processing, and can be easily deposited, e.g. via spinning or evaporation. Benefitting also from the increased absorption mediated by the metals, metal-organic hybrids have been used for a variety of applications, notably in strong coupling ${ }^{25,26}$ and sensing ${ }^{1,27,28}$.

A particularly useful deposition method for the realization of homogenised metamaterials could be the co-evaporation of metals and organic dye in suitable dielectric matrices. In organic electronics, co-evaporation of organic materials and reactive alkali metals is widely used for $\mathrm{n}$-doping of organic semiconductors ${ }^{29,30}$. While noble metals such as $\mathrm{Ag}$ have also been used more recently ${ }^{31}$, no plasmonic interaction has been reported for co-evaporated films so far.

Here we demonstrate the experimental fabrication and characterisation of homogenised metamaterials made by composites of silver and the organic host-guest system formed by the dye 2,3,6,7-tetrahydro-1,1,7,7,-tetramethyl-1H,5H,11H-10-(2-benzothiazolyl)-quinolizino[9,9a,1gh]-coumarin (C545T) doped into a host of 2-methyl-9,10-bis(naphthalen-2-yl)anthracene (MADN). We varied the concentration of silver, extracted the effective permittivity with ellipsometry and measured the fluorescence of films with increasing Ag concentration. We find that the noble nature of Ag leads to the formation of nanoparticles, exhibiting strong localised surface plasmon resonances until a percolation threshold is reached. These findings provide a basis for the development of mixed metal-organic systems and showcase the resulting plasmonic interaction from directly co-evaporated materials. From the analysis we can gather useful parameters and predictions for future gain-compensated plasmonics or ENZ metamaterials.

\section{Results and discussion}

Figure 1 (a) shows the absorption spectrum of an $80 \mathrm{~nm}$ thick film of MADN doped with $10 \mathrm{wt} \%$ of C545T (red line). The spectra show dominant excitonic absorption of the host MADN at $405 \mathrm{~nm}, 383 \mathrm{~nm}$, and $364 \mathrm{~nm}$ as well as the guest C545T at $482 \mathrm{~nm}$ and $459 \mathrm{~nm}$. The photoluminescence (PL) spectrum recorded under excitation at $375 \mathrm{~nm}$ in Fig. 1 (b) shows broad C545T emission peaking at $535 \mathrm{~nm}$, , inidicating an efficient energy transfer from host to guest. For comparison, Fig. 1 (a) also shows the inverse transmission (1-T) of an $80 \mathrm{~nm}$ thick silver film, indicating negligible transmission over the observed spectral range.
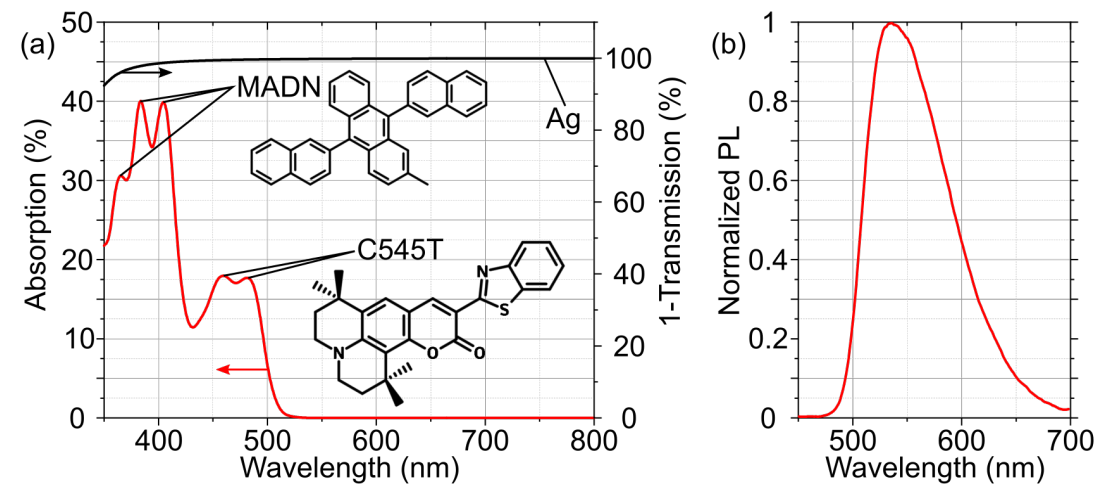
Figure 1: (a) Absorption spectrum of $80 \mathrm{~nm}$ MADN:C545T (red line) and transmission spectrum of $80 \mathrm{~nm}$ Ag (black line). Insets show the chemical structures of MADN and C545T with the black lines indicating their respective main absorption peaks. (b) Photoluminescence spectrum of MADN:C545T, showing exclusive emission from the C545T guest molecules.

During co-evaporation of organic molecules and noble metals, the low chemical reactivity and high surface energy of metal atoms promote the formation of islands or nanoparticles, comparable to the growth of thin metal films on top of organics ${ }^{32}$. While this is often undesired, e.g. for contact electrodes, the resulting plasmonic resonances can also be beneficial for organic optoelectronics, promoting absorption and scattering of light ${ }^{33,34}$. In co-evaporated films of MADN, C545T and Ag at various mixing ratios, we observe the formation of a strong extinction resonance. Looking at the sample absorption of pure organic and mixed films, depicted as 1-transmission spectra in Figure 2, we can identify several regions. In the spectra of the pure organic film (yellow), excitonic absorption decreases transmission as expected, comparable to the absorption spectra in Fig. 1(a). Above $520 \mathrm{~nm}$, transmission is still reduced by $\sim 20 \%$, which can be attributed to reflection of light at the organic-air and organic-substrate interfaces (refractive indices, $n_{\text {org }} \sim 1.75, n_{\text {sub }} \sim 1.52$ ), as the film shows no further extinction above that wavelength. For increasing percentages of $\mathrm{Ag}$, the amount of reflected light at longer wavelengths increases, as could be expected, and a new, strong extinction peak arises between $400 \mathrm{~nm}$ and $500 \mathrm{~nm}$. Depending on size and shape, silver nanoparticles show strong extinction in this spectral region, increasing in intensity and shifting to the red with increasing size. For our data, between $5 \mathrm{vol} \% \mathrm{Ag}$ and $30 \mathrm{vol} \% \mathrm{Ag}$, an increasing extinction of such nanoparticles can be observed. At $50 \mathrm{vol} \% \mathrm{Ag}$, the transmission spectrum changes considerably and resembles the spectrum of pure Ag (compare Fig. 1(a)). This indicates that the percolation threshold of silver inclusions is likely reached here, showing a transition from an insulating film with separated nanoparticles, to a conductive film.

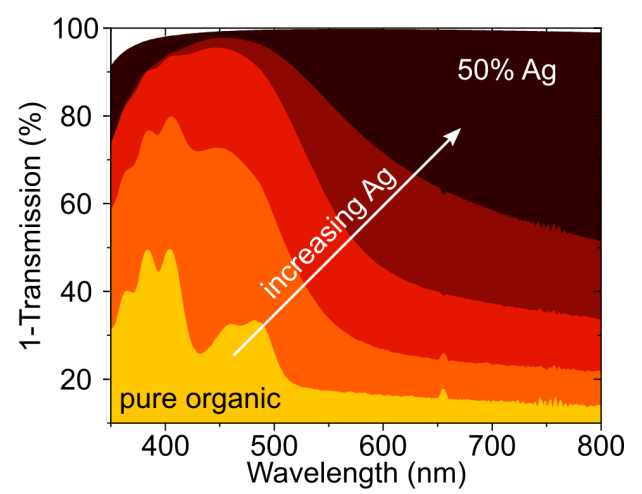

Figure 2: Transmission spectra of MADN:C545T films with increasing concentration of Ag. From yellow to dark red: 0 vol\%, 5 vol\%, 15 vol\%, 30 vol\%, 50 vol\% Ag. With increasing concentration of $\mathrm{Ag}$, strong plasmonic absorption of silver nanoparticles takes over from the highly excitonic spectra of the pure organic layer. At $50 \mathrm{vol} \% \mathrm{Ag}$, the transmission spectrum resembles that of bulk silver (compare Fig. 1 (a)).

While transmission spectra provide a simple and quick method for identifying absorption bands, they cannot distinguish between e.g. absorption and reflection or determine layer thickness. Instead, for a full linear optical characterisation it is important to determine the effective permittivities (or refractive indices) to understand the material more fully and to model the optical response of the homogenized film. There are several models to predict the effective permittivity of a composite layer. At present the most prominent two are the Maxwell-Garnett 
approximation ${ }^{35}$, where spherical inclusions of one material are distributed in the bulk of a second host material, and the Bruggeman model ${ }^{36}$, where both materials are treated as inclusions in an effective medium. The outcome of such modelling is highly dependent on the permittivities of the composite materials as well as the shape and size of the inclusions, which can also change depending on experimental conditions. A convincing prediction can thus only be made when detailed film growth characteristics are known. To avoid these complications, we instead experimentally determined the permittivity for different silver percentages independently.

To achieve this, we performed varying angle spectroscopic ellipsometry (VASE) on films with varying Ag concentration and subsequently determined the imaginary part of the permittivity via modelling and the real part via the Kramers-Kronig relation. Here, films were deposited and evaluated on both glass and crystalline Si substrates and both VASE and transmission measurements were utilised to ensure consistency in modelling.

Figure $3(a)$ and (b) show the resulting permittivity of the ordinary optical axis (extraordinary permittivity is shown in the Supplementary Material, Fig. S1). The pure organic film shows optical anisotropy or birefringence, which in turn continues through all investigated films. Through the analysis of $\varepsilon_{\text {ord }}$, the transition from organic material to metal can be understood. While the pure organic materials show a positive real part of $\varepsilon_{\text {ord }}$, the losses in the imaginary part of $\varepsilon_{\text {ord }}$ indicate excitonic absorption. When adding Ag to the composite film, a strong peak in $\operatorname{Im}\left(\varepsilon_{\text {ord }}\right)$ can be observed, starting at $\sim 450 \mathrm{~nm}$ and shifting towards the red as well as increasing in magnitude with increasing concentration. This additional peak coincides well with the expected behaviour of localized surface plasmons (LSPs) in silver nanoparticles ${ }^{33}$ at the so-called Fröhlich condition ${ }^{37}$, when $\operatorname{Re}\left(\varepsilon_{A g}\right)=-2 \operatorname{Re}\left(\varepsilon_{\text {org }}\right)$. This also suggests that nanoparticles are largely spherical in shape. At $50 \mathrm{vol} \% \mathrm{Ag}$ the behaviour changes drastically, as expected. The extinction peak related to LSPs vanishes and a more continuous behaviour is observed. In addition, the real part of $\varepsilon_{\text {ord }}$ turns negative to further indicate a response closer to bulk silver. From the analysis of the material permittivity, we can draw several conclusions: (i) Silver nanoparticles are formed at concentrations between $5 \mathrm{vol} \%$ and $30 \mathrm{vol} \%$ and their corresponding extinction spectra overlap well with the excitonic absorption of C545T. (ii) Between 30 vol\% and 50 vol\%, the silver particles likely reach percolation threshold, leading to a change from metallic inclusions that cause LSP absorption to a more bulk material response. (iii) In the same range, the real part of the effective permittivity crosses from positive to negative, indicating the possibility of realising ENZ conditions here. However, due to the combination of a metal and a dielectric, high losses are still present.

The formation of silver nanoparticles can be confirmed by scanning electron microscopy (SEM) images of the film surface, as shown in Fig. 3 (c)-(e). From these, it is clear that silver nanoparticles increase both in size and density for increasing silver concentration in the film. 

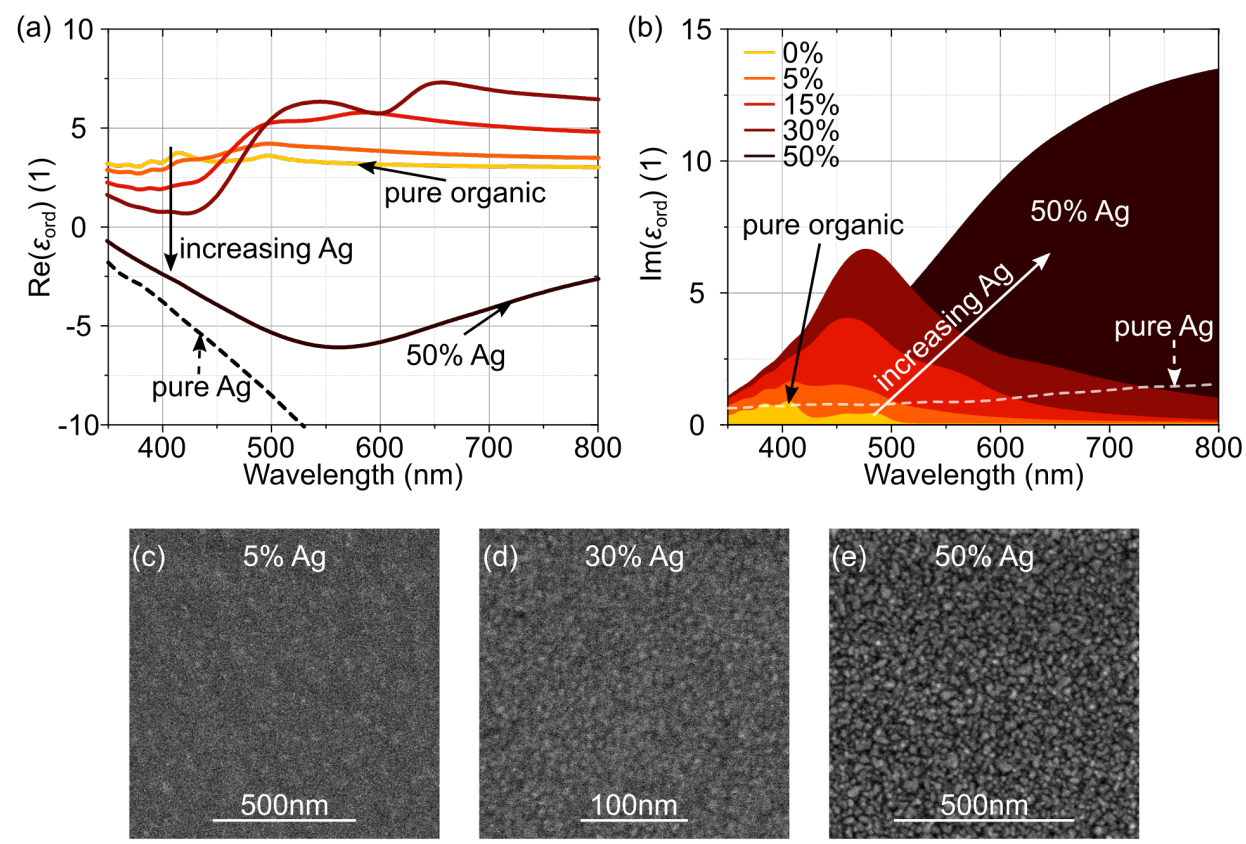

Figure 3: (a),(b) Real (a) and imaginary (b) part of the ordinary permittivity of MADN:C545T films with increasing concentration of Ag. From yellow to dark red: 0 vol\%, 5 vol\%, 15 vol\%, 30 vol\%. 50 vol\% Ag. The dashed black and white lines indicate literature values of bulk $\mathrm{Ag}^{38}$. (c)-(d) SEM images of films containing 5 vol\% (c), 30 vol\% (d), and 50 vol\% (e) of Ag.

As the occurrence of LSPs influences light-matter interaction and thus enhances absorption of light at the peak absorption of the organic molecules, we further investigate the resulting relative PL intensity of the mixed films. Figure 4 shows PL spectra of 5 vol\% to $30 \mathrm{vol} \% \mathrm{Ag}$ films. Here, we observe an increase in PL intensity for up to $15 \mathrm{vol} \% \mathrm{Ag}$ and a subsequent decrease, indicating that the plasmonic interaction can enhance PL by increased interaction with light. At even higher concentrations of Ag PL intensity decreases rapidly (data not shown). The measured samples show no large deviation in ellipsometric response or PL intensity after storage in air for up to three months.
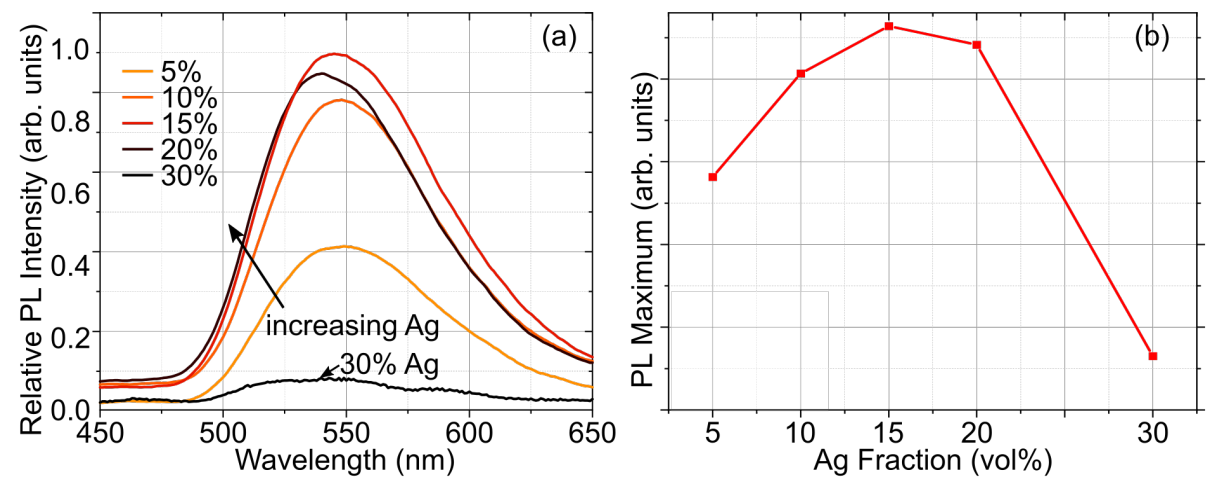

Figure 4: (a) Relative photoluminescence intensity of MADN:C545T films with increasing concentration of Ag. From orange to dark red: 5 vol\%, $10 \mathrm{vol} \%, 15 \mathrm{vol} \%$, and $20 \mathrm{vol} \% \mathrm{Ag}$. $30 \mathrm{vol} \% \mathrm{Ag}$ is shown in black. (b) Relative peak PL intensity for varying Ag concentration.

\section{Conclusion}


In conclusion, we have analyzed the optical properties of co-evaporated metal-organic films of $\mathrm{Ag}$ and dye doped MADN. Through transmission spectroscopy and variable angle spectroscopic ellipsometry, we were able to identify strong extinction resonances in the composite material, a tell-tale sign of localized surface plasmon resonances emanating from silver nanoparticles. The increased light-matter interaction increases the absorbance of the film and in turn leads to an increased photoluminescence intensity despite the presence of additional losses, reaching a maximum for a Ag concentration of $15 \mathrm{vol} \%$. At a ratio between $30 \mathrm{vol} \%$ and $50 \mathrm{vol} \% \mathrm{Ag}$, we observed a percolation threshold and the resulting disappearance of the LSP resonance. In addition, we found that the real part of the effective permittivity switches from positive to negative in this $\mathrm{Ag}$ concentration range. Linking the deposition parameters with the effective optical properties of the deposited film will enable the design and fabrication of gain compensated bulk films with tailored permittivity. To realize epsilon-near-zero metamaterials, the nanoparticle formation should be reduced or suppressed, either via the use of a more reactive metal or via variation of deposition parameters, such as evaporation rate and substrate temperature, both of which are known to allow reduction of the island-like growth of $\mathrm{Ag}$.

\section{Methods}

Sample fabrication: 2,3,6,7-tetrahydro-1,1,7,7,-tetramethyl-1H,5H,11H-10-(2-benzothiazolyl)quinolizino-[9,9a,1gh]-coumarin (C545T) and 2-methyl-9,10-bis(naphthalen-2-yl)anthracene (MADN) are purchased from Lumtec in sublimed grade and used as received. Glass and $\mathrm{Si}$ substrates are cleaned by ultrasonication in Acetone, and 2-propanol and glass substrates are additionally treated by $\mathrm{O}_{2}$ plasma in a plasma asher for $3 \mathrm{~min}$. Thin films are deposited by thermal co-evaporation of host and guest molecules and pure silver in a vacuum chamber (Angstrom EvoVac) at a base pressure of $1 \times 10^{-7}$ mbar. Deposition rates are $\sim 1 \AA / s$ for the host and the deposition rates for the guest and Ag were adjusted according to the desired mixing ratio of each sample.

Sample characterization: Transmission measurements are performed in a 2-beam spectrophotometer (Cary 500). Photoluminescence measurements are taken in a fluorescence lifetime spectrometer (Picoquant Fluotime 250) under excitation by a picosecond pulsed laser at $375 \mathrm{~nm}$ (Picoquant P-C-375). The permittivity is determined from variable angle spectroscopic ellipsometry measurements (VASE, M2000, J.A. Woollam) and subsequent modelling (via CompleteEase software, J.A. Woollam) with an anisotropic Bspline, independently for each sample (representative modelled data is shown in the Supplementary Material, Figure S2). Modelling is performed using transmission and VASE measurement on both glass and $\mathrm{Si}$ Substrates to yield consistent fits to ellipsometric polarization and transmission data. Measurements are repeated after 3 weeks of sample storage in air without encapsulation, with no visible changes in spectra. The scanning electron microscope images are acquired with a Hitachi $\$ 4800$.

\section{Supplementary Material}

Supplementary material showing the extraordinary refractive indices and examples of ellipsometric modelling is available.

\section{Acknowledgements}


A.M. acknowledges funding through an individual fellowship of the Deutsche Forschungsgemeinschaft (No. 404587082). A.D.F. is supported by the European Research Council (ERC) grant AMPHIBIANS (819346). M.C.G. acknowledges funding by the Volkswagen Foundation within project No. 93404 and the ERC via StG ABLASE (640012).

\section{Data availability}

The data that support the findings of this study are openly available in the St Andrews Research Portal at [doi to be set up after review].

\section{References}

${ }^{1}$ K.A. Willets and R.P. Van Duyne, Annu. Rev. Phys. Chem. 58, 267 (2007).

${ }^{2}$ M. Song, D. Wang, S. Peana, S. Choudhury, P. Nyga, Z.A. Kudyshev, H. Yu, A. Boltasseva, V.M. Shalaev, and A. V. Kildishev, Appl. Phys. Rev. 6, (2019).

${ }^{3}$ K. Leosson, T. Nikolajsen, A. Boltasseva, and S.I. Bozhevolnyi, 14, 251 (2006).

${ }^{4}$ P. Berini, Adv. Opt. Photonics 1, 484 (2009).

${ }^{5}$ M.C. Gather, K. Meerholz, N. Danz, and K. Leosson, Nat. Photonics 4, 457 (2010).

${ }^{6}$ I. De Leon and P. Berini, Nat. Photonics 4, 382 (2010).

${ }^{7}$ S. Kéna-Cohen, P.N. Stavrinou, D.D.C. Bradley, and S.A. Maier, Nano Lett. 13, 1323 (2013).

${ }^{8}$ M. Ambati, S.H. Nam, E. Ulin-Avila, D.A. Genov, G. Bartal, and X. Zhang, Nano Lett. 8, 3998 (2008).

9 J. Seidel, S. Grafström, and L. Eng, Phys. Rev. Lett. 94, 177401 (2005).

10 S.I. Azzam, A. V. Kildishev, R.-M. Ma, C.-Z. Ning, R. Oulton, V.M. Shalaev, M.I. Stockman, J.-L. Xu, and X. Zhang, Light Sci. Appl. 9, 90 (2020).

${ }^{11}$ R.-M. Ma, R.F. Oulton, V.J. Sorger, G. Bartal, and X. Zhang, Nat. Mater. 10, 110 (2011).

${ }^{12}$ M.T. Hill, M. Marell, E.S.P. Leong, B. Smalbrugge, Y. Zhu, M. Sun, P.J. van Veldhoven, E.J. Geluk, F. Karouta, Y.-S. Oei, R. Nötzel, C.-Z. Ning, and M.K. Smit, Opt. Express 17, 11107 (2009).

${ }^{13}$ M.C. Gather, K. Meerholz, N. Danz, and K. Leosson, Nat. Photonics 4, 457 (2010).

${ }^{14}$ H. Wu, Y. Gao, P. Xu, X. Guo, P. Wang, D. Dai, and L. Tong, Adv. Opt. Mater. 7, 1900334 (2019).

${ }^{15}$ S.I. Azzam, A. V. Kildishev, R.-M. Ma, C.-Z. Ning, R. Oulton, V.M. Shalaev, M.I. Stockman, J.-L. Xu, and X. Zhang, Light Sci. Appl. 9, 90 (2020).

${ }^{16}$ A. Alù, M.G. Silveirinha, A. Salandrino, and N. Engheta, Phys. Rev. B 75, 155410 (2007).

${ }^{17}$ A. Salandrino and N. Engheta, Phys. Rev. B - Condens. Matter Mater. Phys. 74, 1 (2006).

18 J. Kim, A. Dutta, G. V. Naik, A.J. Giles, F.J. Bezares, C.T. Ellis, J.G. Tischler, A.M. Mahmoud, H. Caglayan, O.J. Glembocki, A. V. Kildishev, J.D. Caldwell, A. Boltasseva, and N. Engheta, Optica 3, 339 (2016).

${ }^{19}$ L. Caspani, R.P.M. Kaipurath, M. Clerici, M. Ferrera, T. Roger, J. Kim, N. Kinsey, M. Pietrzyk, A. Di Falco, V.M. Shalaev, A. Boltasseva, and D. Faccio, Phys. Rev. Lett. 116, 
233901 (2016).

${ }^{20}$ M. Clerici, N. Kinsey, C. DeVault, J. Kim, E.G. Carnemolla, L. Caspani, A. Shaltout, D. Faccio, V. Shalaev, A. Boltasseva, and M. Ferrera, Nat. Commun. 8, 15829 (2017).

${ }^{21}$ E.G. Carnemolla, L. Caspani, C. DeVault, M. Clerici, S. Vezzoli, V. Bruno, V.M. Shalaev, D. Faccio, A. Boltasseva, and M. Ferrera, Opt. Mater. Express 8, 3392 (2018).

${ }^{22}$ C. Rizza, A. Di Falco, and A. Ciattoni, Appl. Phys. Lett. 99, 221107 (2011).

${ }^{23}$ S. Campione, M. Albani, and F. Capolino, Opt. Mater. Express 1, 1077 (2011).

${ }^{24}$ L. Sun, X. Yang, and J. Gao, Appl. Phys. Lett. 103, 201109 (2013).

${ }^{25}$ F. Benz, M.K. Schmidt, A. Dreismann, R. Chikkaraddy, Y. Zhang, A. Demetriadou, C. Carnegie, H. Ohadi, B. de Nijs, R. Esteban, J. Aizpurua, and J.J. Baumberg, Science (80-. ). 354, 726 (2016).

${ }^{26}$ R. Chikkaraddy, B. de Nijs, F. Benz, S.J. Barrow, O.A. Scherman, E. Rosta, A. Demetriadou, P. Fox, O. Hess, and J.J. Baumberg, Nature 535, 127 (2016).

${ }^{27}$ M. Lu, H. Zhu, C.G. Bazuin, W. Peng, and J.-F. Masson, ACS Sensors 4, 613 (2019).

${ }^{28}$ A. Amirjani and D.H. Fatmehsari, Talanta 176, 242 (2018).

${ }^{29}$ R. Meerheim, K. Walzer, M. Pfeiffer, and K. Leo, Appl. Phys. Lett. 89, 061111 (2006).

${ }^{30}$ C.-M. Keum, N.M. Kronenberg, C. Murawski, K. Yoshida, Y. Deng, C. Berz, W. Li, M. Wei, I.D.W. Samuel, and M.C. Gather, Adv. Opt. Mater. 6, 1800496 (2018).

${ }^{31}$ Z. Bin, G. Dong, P. Wei, Z. Liu, D. Zhang, R. Su, Y. Qiu, and L. Duan, Nat. Commun. 10, 866 (2019).

32 S. Schubert, J. Meiss, L. Müller-Meskamp, and K. Leo, Adv. Energy Mater. 3, 438 (2013).

${ }^{33}$ S.H. Jeong, H. Choi, J.Y. Kim, and T.W. Lee, Part. Part. Syst. Charact. 32, 164 (2015).

${ }^{34}$ S.W. Baek, J. Noh, C.H. Lee, B. Kim, M.K. Seo, and J.Y. Lee, Sci. Rep. 3, 1 (2013).

${ }^{35}$ V.A. Markel, J. Opt. Soc. Am. A 33, 1244 (2016).

${ }^{36}$ T.C. Choy, Effective Medium Theory: Principles and Applications (Oxford University Press, 2015).

${ }^{37}$ K.L. Kelly, E. Coronado, L.L. Zhao, and G.C. Schatz, J. Phys. Chem. B 107, 668 (2003).

${ }^{38}$ E.D. Palik, Handbook of Optical Constants of Solids (Academic press, 1998). 